\title{
População e território de forrageamento de uma colônia de Heterotermes tenuis (Hagen) (Isoptera, Rhinotermitidae)
}

\author{
Célia Regina Rodrigues de Camargo-Dietrich \& Ana Maria Costa-Leonardo
}

Departamento de Biologia, Instituto de Biociências, Universidade Estadual Paulista. Caixa Postal 199, 13506-900 Rio Claro, São Paulo, Brasil. E-mail amcl@rc.unesp.br

\begin{abstract}
Population and foraging territory of a colony of Heterotermes tenuis (Hagen) (Isoptera, Rhinotermitidae). Heterotermes tenuis (Hagen, 1858) is a subterranean termite that cause damage in forests, crops and urban areas. The population and foraging territory of an urban colony were determined using triple markrelease-recapture technique. Foraging population was estimated in 134,583 $\pm 5,803$ individuals. The foraging territory encompassed an area of $175,5 \mathrm{~m}^{2}$ and the maxim linear foraging distance was $36 \mathrm{~m}$.
\end{abstract}

KEY WORDS. Isoptera, Rhinotermitidae, Heterotermes tenuis, population, territory.

A população do cupim Neotropical Heterotermes tenuis (Hagen, 1858) é composta por indivíduos de pequeno porte, com soldados dimórficos (grandes e pequenos) e apresenta hábitos subterrâneos (CONSTANTINo 1991, FonTES 1995). Causa danos em ambientes agrícolas, florestais e urbanos, mas devido à sua natureza críptica, pouco se conhece sobre a sua biologia e os escassos estudos realizados sobre essa espécie se restringem à ocorrência em determinadas culturas ou no próprio meio urbano (Araujo 1958, 1986, Pizano \& Fontes 1986, Mill 1991, 1992, Camargo-Dietrich \& Costa-Leonardo 2000).

As colônias de cupins subterrâneos geralmente estão localizadas sob o solo e essa característica dificulta o estudo demográfico e a análise do comportamento de forrageamento de suas populações. Uma das alternativas para o estudo dessas espécies é o emprego da técnica de marcação e recaptura, adaptada para populações de cupins. A vantagem dessa técnica é que ela não é destrutiva, permitindo o estudo das mesmas populações por um período longo e podendo ser usada em ambientes urbanos, onde o forrageamento e os ninhos de cupins ocorrem sob lajes de concreto, pavimentos de asfalto e dentro de edificações (Begon 1979, Su \& SCHEFFrahn 1988, GraCE 1990; Jones 1990a, b, Su et al. 1993, HaAgsma \& Rust 1995, CostaLeonardo 1996, Forchler \& Townsed 1996, Costa-Leonardo \& Camargo-Dietrich 1999, Costa-Leonardo 2002).

Nesse trabalho utilizou-se a técnica de marcação e recaptura para estimar a população e o território de forrageamento de uma colônia de $H$. tenuis localizada em área urbana.

\section{MATERIAL E MÉTODOS}

O estudo foi realizado em uma colônia de $H$. tenuis localizada no campus da Universidade Estadual Paulista (UNESP) de Rio Claro $\left(22^{\circ} 55^{\prime} \mathrm{S}, 4^{\circ} 31^{\prime} \mathrm{W}\right.$ e altitude de $576 \mathrm{~m}$ ).
Ao redor do prédio do Biotério, numa área aproximada de $1.400 \mathrm{~m} 2$, foram instaladas 28 estacas de madeira (Pinus sp.) de $5 \times 5 \times 25 \mathrm{~cm}$ e 55 armadilhas de papelão corrugado, para localizar pontos de infestação de $H$. tenuis, uma vez que foi observada a presença dessa espécie no interior de duas salas do prédio em estudo (Fig. 1). Nos pontos de infestação foram estabelecidas as estações de monitoramento, que consistiram de canos de PVC de $12 \mathrm{~cm}$ de diâmetro por $20 \mathrm{~cm}$ de comprimento, enterrados no solo. No interior desses canos foram colocadas armadilhas de papelão corrugado de $10 \mathrm{~cm}$ de diâmetro por $15 \mathrm{~cm}$ de altura, envoltas por meias embalagens plásticas descartáveis de refrigerante e para protegê-las na parte superior dos canos foi colocado papel alumínio. Nos dois pontos de infestação localizados no interior das salas do Biotério foram colocadas somente as armadilhas, que também serviram de estações de monitoramento.

A estimativa da população e do território de forrageamento da colônia foi efetuada pelo método de marcação e recaptura (BEGON 1979), adaptado para populações de cupins por Su \& SCHEFFrahn (1988).

Para a aplicação da técnica de marcação e recaptura, foram realizadas inspeções e reposições de todas as armadilhas das estações de monitoramento. A estação número 6 (Fig. 1) foi escolhida como ponto de partida do estudo porque ela apresentava o maior número de indivíduos. Os 1.700 operários provenientes dessa armadilha foram conduzidos para o laboratório, colocados em placas de Petri contendo papéis-filtro $(9$ $\mathrm{cm}$ de diâmetro) corados com a solução de Azul de Nilo a $0,1 \%$ e deixados para se alimentarem por um período de sete dias. Após esse período, os operários sobreviventes marcados (1.435) foram devolvidos para a respectiva estação de monitoramento.

Todas as armadilhas foram inspecionadas após sete dias e, naquelas que apareceram os indivíduos corados, foi deter- 
minado o número de corados e não corados. Esses indivíduos foram colocados novamente para se alimentarem, por um período de sete dias, com papel-filtro corado e foram liberados nas respectivas estações de monitoramento em que foram coletados, repetindo-se o ciclo. No total, foram realizados três ciclos de marcação e recaptura que envolveram 42 dias.

Os cupins das estações de monitoramento onde apareceram operários corados foram considerados pertencentes à mesma colônia, e o território de forrageamento dessa colônia foi delineado pelos pontos onde apareceram os indivíduos corados durante os três ciclos de marcação e recaptura.

A estimativa da população de forrageamento $(\mathrm{N})$ foi realizada pelo método da média ponderada, onde:

$$
\mathrm{N}=\left(\sum \mathrm{Mi} \cdot \mathrm{ni}\right) /\left[\left(\sum \mathrm{mi}\right)+1\right]
$$

sendo: (Mi ) número total de indivíduos marcados, até o iésimo ciclo; (ni) número de indivíduos recapturados; (mi) número de indivíduos corados, entre os recapturados.

A esse método foi associado o cálculo do erro padrão (EP) onde:

$$
E P=N \cdot \sqrt{\left[1 /\left(\sum \mathrm{mi}+1\right)\right]+\left[2 /\left(\sum \mathrm{mi}+1\right)^{2}\right]+\left[6 /\left(\sum \mathrm{mi}+1\right)^{3}\right]}
$$

O peso fresco do operário foi determinado pela média do peso de 50 indivíduos. A biomassa forrageira foi calculada multiplicando-se esse valor pelo número estimado de forrageiros na população.

\section{RESULTADOS E DISCUSSÃO}

Os números de operários capturados que foram marcados e liberados, os recapturados e os marcados entre os recapturados, durante o programa triplo de marcação e recaptura, estão representados na tabela I.

A aplicação da técnica de marcação e recaptura mostrou a presença de duas colônias próximas ao prédio do Biotério (Fig. 1). A primeira colônia está localizada nas estações de monitoramento $1,2,3$, 4 e 5 e a segunda nas estações $6,7,8$, 9 e 10 , a qual foi utilizada para o estudo da estimativa da população e do território de forrageamento.

A população de forrageamento da colônia estudada foi de $134.583 \pm 5.803$ indivíduos, valor inferior ao daquelas citadas por CoSTA-LEONARDO (2002), que foram respectivamente, 389.313 $\pm 14.907,641.600 \pm 12.127$ e $265.589 \pm 12.635$ indivíduos, em três colônias de $H$. tenuis também localizadas no campus da UNESP. O território de forrageamento, $175,5 \mathrm{~m}^{2}$, foi maior do que os encontrados no referido trabalho, 93,6, 70 e $131 \mathrm{~m}^{2}$, respectivamente. A distância máxima percorrida pelos forrageiros da colônia estudada foi de $36 \mathrm{~m}$, valor também superior ao das três colônias citadas anteriormente, que foram respectivamente 14,28 e $19 \mathrm{~m}$. A biomassa forrageira estimada em $0,20 \mathrm{~kg}$ foi inferior ao das três colônias $(0,77,1,17$ e $0,51 \mathrm{~kg})$.

As diferenças entre as populações de forrageamento, observadas nas colônias de $H$. tenuis em estudo e aquelas acima citadas, podem estar relacionadas à idade das mesmas. Enquanto que, as variações das áreas de forrageamento devem estar relacionadas com a disponibilidade de alimento e presença de competidores e predadores.

Em um estudo realizado por Almeida et al. (1999), para determinar a área de forrageamento do cupim $H$. tenuis em cana-

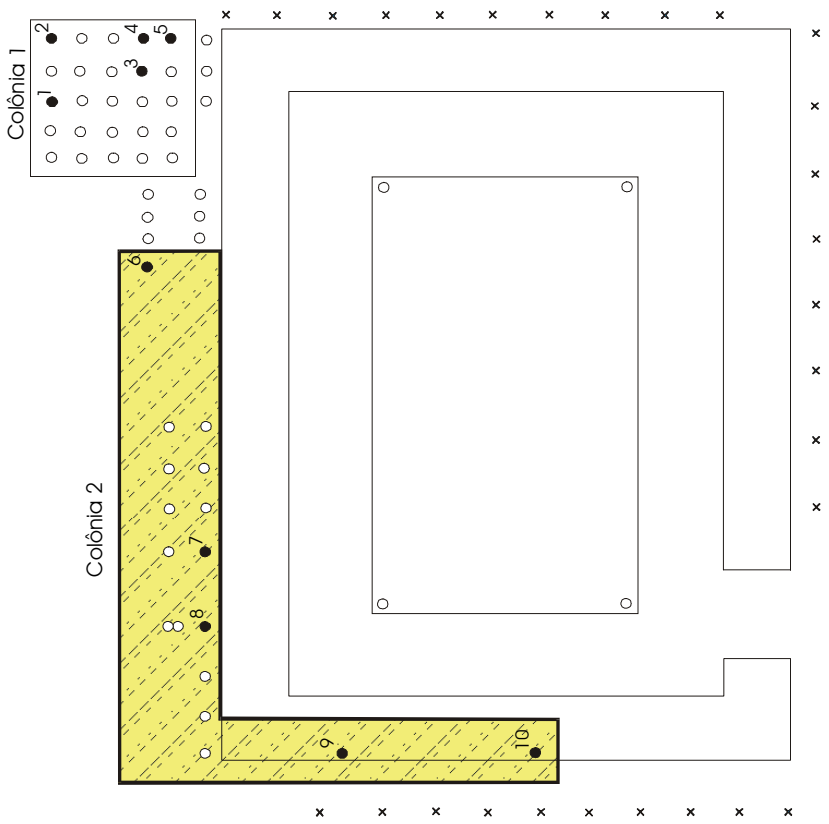

Figura 1. Território de forrageamento (área sombreada) de uma colônia de Heterotermes tenuis. Os círculos cheios indicam as estações de monitoramento, os círculos vazios indicam as armadilhas de papelão corrugado e os ( $\mathrm{x}$ ), as estacas de Pinus sp. instaladas. Escala: 1:250.

de-açúcar, utilizando armadilhas de papelão marcadas com o radioisótopo ${ }^{32} \mathrm{P}$, foram encontradas diversas colônias forrageando numa mesma área e, essa pode variar de 3 a $1.250 \mathrm{~m}^{2}$. O território de forrageamento da colônia em estudo $\left(175,5 \mathrm{~m}^{2}\right)$ se encontra dentro dos valores citados pelos autores acima, apesar dos mesmos não considerarem o território individual de cada colônia.

A população de forrageamento da colônia de $H$. tenuis estudada está em conformidade com os valores obtidos para algumas espécies de cupins da família Rhinotermitidae. JonEs (1990b) estudou populações de Heterotermes aureus (Snyder, 1920) no Arizona (E.U.A.) e verificou que elas variavam de $44.868 \pm 4.946$ a $301.189 \pm 19.935$ indivíduos. HAagsma \& RusT (1995) estimaram as populações de Reticulitermes hesperus Banks, 1920 em área urbana e natural, na Califórnia (E.U.A.), e verificaram que elas variavam de $85.752 \pm 39.894$ a $830.581 \pm$ 224.860 indivíduos em área urbana e de $78.930 \pm 18.692$ a $103.758 \pm 46.931$ em área natural.

Na espécie exótica Coptotermes havilandi Holmgren, 1911 pertencente à família Rhinotermitidae e considerada praga em áreas urbanas do Estado de São Paulo, são encontradas colônias com populações superiores à de $H$. tenuis estudada. CostaLEONARDO \& CAMARGO-Dietrich (1999) estimaram a população de uma colônia de C. havilandi, localizada em Rio Claro, São Paulo, em $729.918 \pm 33.740$ indivíduos. Em outro estudo realizado em Rio Claro, as populações de duas colônias de $C$. havilandi foram estimadas em $575.721 \pm 9.330$ e $737.545 \pm$ 14.834 indivíduos (Costa-Leonardo 2002). 
Tabela I. Número de operários capturados, marcados e liberados, de recapturados e de marcados entre os recapturados durante o programa triplo de marcação e recaptura em uma colônia de Heterotermes tenuis localizada em área urbana. Os números entre parênteses representam as estações de monitoramento onde os indivíduos foram liberados e recapturados.

\begin{tabular}{rrlrr}
\hline Ciclos & Operários capturados, marcados e liberados & \multicolumn{2}{c}{ Operários recapturados } & Operários marcados e recapturados \\
\hline 1 & 1.435 & $(6)$ & 4.695 & $(6,7,9)$ \\
2 & $4.361 \quad(6,7,9)$ & $4.890 \quad(6,7,8,9,10)$ & 213 \\
3 & $4.056 \quad(6,7,8,9,10)$ & $3.816 \quad(6,7,8,9,10)$ & 298 \\
\hline Total & 9.852 & 13.401 & 539 \\
\hline
\end{tabular}

A distância máxima $(36 \mathrm{~m})$ percorrida pelos forrageiros de $H$. tenuis da colônia em estudo está dentro dos valores descritos para outras espécies de cupim, pertencentes à mesma família. GRACE (1990) avaliou a distância máxima percorrida por Reticulitermes flavipes Kollar, 1837 e verificou que os valores variaram de 16 a $46 \mathrm{~m}$, enquanto que $\mathrm{Su}_{\mathrm{u}}$ (1994) encontrou uma variação de 9 a $71 \mathrm{~m}$ para a mesma espécie. CosTA-LEONARDo $\&$ CAMARGO-Dietrich (1999) encontraram o valor de $33 \mathrm{~m}$ como sendo a distância máxima percorrida pelos forrageiros da espécie C. havilandi.

A metodologia de captura e recaptura mostrou-se viável para o estudo não destrutivo de populações de $H$. tenuis e pode ser considerada uma importante ferramenta para avaliação de populações e territórios de forrageamento, antes e após a aplicação de um método de controle.

\section{AGRADECIMENTOS}

Os autores agradecem ao CNPq pelo apoio financeiro.

\section{REFERÊNCIAS BIBLIOGRÁFICAS}

AlmeidA, J.E.M.; S.B. Alves \& J.M. WALDER. 1999. Tamanho da área de forrageamento do cupim subterrâneo Heterotermes tenuis em cana-de-açúcar. Scientia Agricola, Piracicaba, 56 (2): 1-8.

ARAujo, R.L.1958. Contribuição à biogeografia dos térmitas de são Paulo, Brasil, Insecta-Isoptera. Arquivos do Instituto Biológico, São Paulo, 25: 185-217.

. 1986. Térmitas prejudiciais às madeiras, p. 100-123. In: F. A.M. Mariconi; A.P.L. Zamith; R.L. Araujo; A.M. Oliveira Filho \& R. Pinchin (Ed.). Inseticidas 3 e seu emprego no combate às pragas. São Paulo, Nobel, Tomo III, $2^{\mathrm{a}}$ ed., 246p.

BEGON, M. 1979. Investigating Animal Abundance: capturerecapture for biologist. London: Edward Arnold, 97p.

Camargo-Dietrich, C.R.R. \& A. M. Costa-Leonardo. 2000. Comportamento intra-específico do cupim Heterotermes tenuis (Hagen) (Isoptera, Rhinotemitidae) em condições de laboratório. Revista Brasileira de Zoologia, Curitiba, 17 (2): 421-427.

Constantino, R. 1991. Termites (Isoptera) from the lower Japurá River, Amazonas State, Brazil. Boletim do Museu Paraense Emílio Goeldi, série Zoologia, Belém, 7 (2): 189-224.

Costa-Leonardo, A.M. 1996. Métodos para a coleta e estudo de cupins subterrâneos. Revista de Agricultura, Piracicaba, 71 (3): 337-345.

2002. Cupins-praga: Morfologia, biologia e controle. Rio Claro, Divisa, 128p.

Costa-Leonardo, A.M. \& C.R.R. Camargo-Dietrich. 1999. Terri- tório e população de forrageio de uma colônia de Coptotermes havilandi (Isoptera: Rhinotermitidae) em meio urbano. Arquivos do Instituto Biológico, São Paulo, 66 (2): 99-105.

FonTeS, L.R. 1995. Sistemática geral de cupins, p. 11-17. In: E. BERTI Filho \& L.R. Fontes (Eds). Alguns aspectos atuais da biologia de cupins. Piracicaba, Fundação de Estudos Agrários Luiz de Queiroz-FEALQ, 183p.

ForsChleR, B.T. \& M. L. TownsEnd. 1996. Mark-release-recapture estimates of Reticulitermes spp. (Isoptera: Rhinotermitidae) colony foraging populations from Georgia, U.S.A. Environmental Entomology, College Park, 25 (5): 953-962.

Grace, J.K. 1990. Mark-recapture studies with Reticulitermes flavipes (Isoptera: Rhinotermitidae). Sociobiology, Chico, 16 (3): 297-303.

HaAgsma, K.A. \& M.K. Rust. 1995. Colony size estimates, foraging trends, and physiological characteristics of the Western subterranean termite (Isoptera: Rhinotermitidae). Environmental Entomology, College Park, 24 (6): 1520-1528.

JoNEs, S.C. 1990a. Delineation of Heterotermes aureus (Isoptera; Rhinotermitidae) foraging territories in a Sonoran desert grassland. Environmental Entomology, College Park, 19 (4): 1047-1054.

. 1990b. Colony size of the desert subterranean termite Heterotermes aureus (Isoptera: Rhinotermitidae). Southwestern Naturalist, College Station, 35 (3): 285-291.

MiLl, A.E. 1991. Termites as structural pests in Amazonia, Brazil. Sociobiology, Chico, 18 (2): 339-348.

. 1992. Termites as agricultural pests in Amazonia, Brazil. Outlook on Agriculture, Bracknel, 21 (1): 41-46.

Pizano, M.A. \& L.R. Fontes. 1986. Ocorrência de Heterotermes tenuis (Hagen, 1858) e H. longiceps (Snyder,1924) (Isoptera, Rhinotermitidae) atacando cana-de-açúcar no Brasil. Brasil Açucareiro, Rio de Janeiro, 104 (3/4): 29.

Su, N-Y. 1994. Field evaluation of a hexaflumuron bait for population suppression of subterranean termites (Isoptera: Rhinotermitidae). Journal of Economic Entomology, College Park, 87(2): 389-397.

Su, N-Y \& R.H. SCheffrahn. 1988. Foraging population and territory of the formosan subterranean termite (Isoptera: Rhinotermitidae) in an urban environment. Sociobiology, Chico, 14 (2): 353-359.

Su, N-Y; P.M. BAN \& R.H. SCHEFFRAHN. 1993. Foraging populations and territories of the eastern subterranean termite (Isoptera: Rhinotermitidae) in Southeastern Florida. Environmental Entomology, College Park, 22, n. (5): 1113-1117.

Recebido em 30.IX.2002; aceito em 11.VIII.2003.

Revista Brasileira de Zoologia 20 (3): 397-399, setembro 2003 